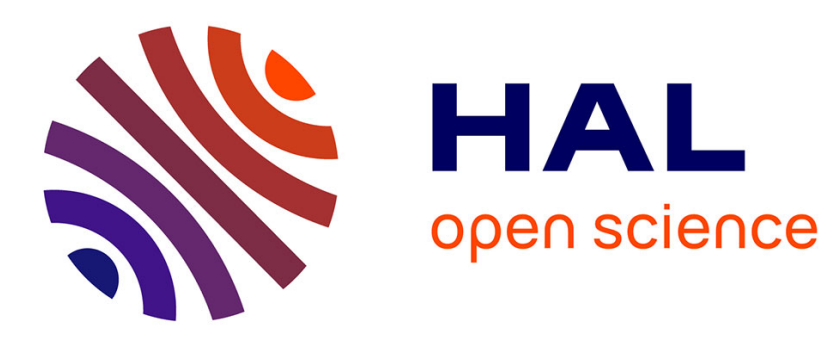

\title{
GNSS Accuracy enhancement based on pseudo range error estimation in an urban propagation environment
}

Juliette Marais, Donnay Fleury Nahimana, Nicolas Viandier, Emmanuel Duflos

\section{- To cite this version:}

Juliette Marais, Donnay Fleury Nahimana, Nicolas Viandier, Emmanuel Duflos. GNSS Accuracy enhancement based on pseudo range error estimation in an urban propagation environment. Expert Systems with Applications, 2013, 40 (15), pp 5956-5964. 10.1016/j.eswa.2013.05.026 . hal-00853813

\section{HAL Id: hal-00853813 https://hal.science/hal-00853813}

Submitted on 28 Oct 2013

HAL is a multi-disciplinary open access archive for the deposit and dissemination of scientific research documents, whether they are published or not. The documents may come from teaching and research institutions in France or abroad, or from public or private research centers.
L'archive ouverte pluridisciplinaire HAL, est destinée au dépôt et à la diffusion de documents scientifiques de niveau recherche, publiés ou non, émanant des établissements d'enseignement et de recherche français ou étrangers, des laboratoires publics ou privés. 


\title{
GNSS Accuracy enhancement based on pseudo range error estimation in an urban propagation environment
}

\author{
J. Marais ${ }^{a, b}$, D.-F. Nahimana ${ }^{a, b}$, N. Viandier ${ }^{a, b}$, E. Duflos ${ }^{a, c, d}$ \\ ${ }^{a}$ Univ Lille Nord de France - F-59000 Lille, France \\ b IFSTTAR, LEOST, French institute of science and technology for transport, development and networks, BP 70317, \\ 20 rue Elisée Reclus, 59655 Villeneuve d'Ascq Cedex, France \\ ${ }^{c}$ Ecole Centrale de Lille, LAGIS UMR CNRS 8219, Cite Scientifique, BP48, 59651 Villeneuve d'Ascq Cedex, France \\ 'INRIA Lille Nord-Europe, SequeL Project Team, Parc Scientifique de la Haute Borne, 40, avenue Halley, Bât.A, \\ Park Plaza, 59650 Villeneuve d'Ascq, France
}

Corresponding author: Juliette Marais, IFSTTAR LEOST, 20 rue E. Reclus, BP317, 59666 Villeneuve d'Ascq cedex, email:juliette.marais@ifsttar.fr, tel: +33.3.20.43.84.95, Fax: +33.3.20.43.83.59

\begin{abstract}
For new ITS applications, positioning solutions will require to be more accurate and available. The most common technique used today is composed of a GPS receiver, sometimes aided by other sensors. GPS, and GNSS in general, suffer from masking effects and propagation disturbances in urban areas that cause biases on pseudo range measurements. Mitigation solutions sometimes propose to detect and exclude outliers but in land transportation applications, such a decision reduces dramatically the service availability and thus, the interest of satellite-based solutions. In order to optimize the use the satellites received, we propose a new positioning algorithm based on signals only with pseudo range error modeling in association with an adapted filtering process. The model and the filter have been validated with simulation data performed along an urban bus line and have shown that both positioning error and availability can be improved. Along the trajectory tested, the mean accuracy has been reduced from $5.3 \mathrm{~m}$ with a classical filter to $2.6 \mathrm{~m}$ with our algorithm with $89 \%$ of the points more accurate than 5 meters instead of $64 \%$ before.
\end{abstract}

Index Terms-GNSS, signal processing, error model, localization, particle filter, Jump Markov System

\section{INTRODUCTION}

\subsection{ITS (Intelligent Transport Systems) applications}

Today most of the ITS rely on a positioning function. Indeed, as stressed in (Ochieng and Sauer, 2002), many transport user groups are customers of positioning information: travel and traffic management, public transportation or electronic payments for example. GPS (Global Positioning System) and other GNSS (Global Navigation Satellite System) are excellent candidates for such applications because the use of the infrastructure is free of charges and because of their performances. However, one of the main drawbacks of GNSS is unavailability of the signals that occur in densely built areas, and the performance degradations caused by multipath or Non-Line of Sight (NLOS) signals that reduces their capacity to reach the RNP (Required Navigation Performance) in terms of accuracy and availability.

To reach better performances, several technical solutions can be studied in compatibility with the operational constraints that include: the cost of the localization system has to be minimized as much as possible.

Most of the solutions already deployed are thus based on the use of GNSS and inertial sensors. For road applications accuracy can also be reduced by map-matching algorithms that always place the vehicle's position on a pre-recorded infrastructure. Such solutions are today convincing for car navigation where the driver decides where he wants to go or for most of the advanced transport telematics applications characterized in (Quddus, 06) in which accuracy is required in the levels of 5-10 meters (announcement of bus stops) or even 10-30 meters (accident management or automatic vehicle location). But in driver assistance services such as collision avoidance support systems, where better accuracy is required to estimate TTC (Time To Collision) and TTR (Time To React), typical navigation subsystems based on a combination of motion and GNSS sensors are not accurate enough (Santa et al., 2009). Furthermore, future applications will be geared towards complete guidance of the vehicle, vehicle-withoutdriver, trains of trucks etc for which position must be accurate enough to distinguish the track of circulation and short distances between the vehicle and the surrounding obstacles (less than $5 \mathrm{~m}$ ). 
Expert Systems with Applications, Volume 40, Issue 15, 1 November 2013, Pages 5956-5964 http://dx.doi.org/10.1016/j.eswa.2013.05.026 OElsevier

EGNOS, the European satellite-based augmentation system could enhance accuracy but suffers from poor availability in urban areas due to their low elevation angle in Europe (Kovár et al., 2005) (Obst et al., 2011) and will offer poor augmentations.

\subsection{Problem statement}

GNSS position computation relies on satellite signal TOA (Time Of Arrival) processed in a classical filtering method usually a Kalman or Extended Kalman filter where the observation noises are assumed white and Gaussian. In the case of LOS (Line Of Sight) signals, this assumption is correct. However in harsh environments, where signals can be reflected by obstacles (buildings, trees...), a bias is induced in the pseudo range, in particular when the direct path of the signal is not available (Jiang, 2012). The noise becomes non-stationary and the white Gaussian assumption is no longer verified. In such a case, the pseudo range error can then be considered following a Normal law, which parameters vary versus time. The use of classical methods thus creates supplementary positioning errors.

The literature is abundant on multipath mitigation techniques in such environments (Wang, 2012). The aim is to detect them to enhance accuracy (Lentmaier et al., 2008). Different techniques are developed among which exclusion procedures to avoid using corrupted data (Pervan et al., 1996). NLOS detection remains however a critical research axis before exclusion. A usable criterion can be C/NO (Carrier to Noise Ratio) or elevation angle (Wang et al., 2007) but the limits of exclusion in land transportation environment are related to satellite visibility, which is often weak. Excluding satellites in the position estimation process can then create more positioning error (outliers) and imply large unavailability periods.

\subsection{Our proposal}

The solution presented in this paper uses GNSS signals only, without any supplementary sensors. The goal is to improve as much as possible the GNSS solution by using all the pseudo ranges available in order to offer both availability and accuracy. Then, it will be possible to add low cost supplementary sensors to reach the required performance if necessary. In classical receiver architecture, we propose a new position solution computation based on the satellite states of reception knowledge.Some of the ideas developed in this paper have been initiated during the ANGO project. Its goal was to provide, to an urban bus driver, accurate and secure longitudinal positioning information all along the route of the vehicle for Advanced Driver Assistance System that aims at improving fuel consumption, comfort and maintenance. The required accuracy for longitudinal positioning is equal to $5 \mathrm{~m}$ with a reliability level depending of the application (Marais and Pouyet, 2007).

In a simulation study, (Nahimana et al., 2007) developed new algorithms based on the estimation of both the state vector and the observation noise according to the reception state of each satellite that determines the noise type to use in a particle filter. Thus the reception state determines the noise kind to apply in a particle filter in order to estimate the vector state.

In this paper, we associate this reception state estimation with an error distribution in order to enhance the filtering process and to increase positioning accuracy. As justified in the introduction, the solution developed in this paper relies on measurements performed by a low cost GPS receiver without any supplementary sensor. Indeed, the work consists in improving as far as possible the GPS receiver efficiency (increase accuracy and availability) before adding supplementary sensors. To solve the unavailability of the system when less than 4 satellites can be received is out of our scope.

In this paper, we will first describe error distribution modeling before developing their use in the filtering process. The results section will illustrate the algorithm efficiency both on simulated and measured GPS data. The conclusion will open on perspectives to this work.

\section{ERROR MODELING}

\subsection{Reception states modeling}

In an urban environment, different satellite signal reception states occur according to the relative positions of satellites and obstacles and according to the nature of obstacles.

Indeed, since the position given by a satellite-based system relies on TOA, the surroundings of the antenna play a major role in the measurement quality. Several events may appear. The best measurement will occur when the direct reception of the signal is available (case LOS) as in obstacles-free environments (antenna is placed on a building roof). In land transportation applications, this direct signal often goes with echoes (two or more) of the same signal caused by the surrounding surfaces (ground, buildings, cars, vegetation...). This phenomenon is named multipath. The received signal is the sum of the different signals received. The range measurement error depends on the strength of each received signal and the delay between them. The receiver architecture, with different signal 
processing stages, conditions the multipath impact on the pseudo-range estimation. Theoretical analysis of multipath errors usually assumes the presence of the direct path, in order to analyze the error depending on the relative power, code delay and phase delay of reflected signals with respect to the direct one. After corrections of the common mode errors (atmospheric delays or clock errors), the residual error observed in LOS is usually reduced as a Gaussian zero centered error.

Because of masking obstacles happening in the LOS between the receiver and the satellite, the signal can be received with no direct ray. In the following, this particular case of multipath will be named "alternate path". In this context, the error model will be biased.

As used in telecommunication studies (Perez-Fontan et al., 2001), we consider three reception states which are:

- The Blocked state: when the receiver does not detect any signal or when the shadowing conditions are too intense to allow the receiver to use the signal.

- The Direct state: when the signal reaches the antenna in LOS. In this state we consider that noise density is white Gaussian. The correlation peak can be distorted because of multipath but, in this paper, we will assume that correlators are capable of extracting the correct, or at least a minimized, propagation delay from it.

- The Masked or Alternate state: when the signal cannot reach the antenna in LOS but after one or many reflections (NLOS). In this state, noise density is not centered Gaussian and the signal power is attenuated because of the reflection.

\subsection{Reception states detection}

As developed in (Marais et al., 2005), C/N0 (Carrier-to-Noise ratio) can illustrate state variations versus time. Figures 1 and 2 present respectively the C/NO variations for one given satellite versus time and the C/NO histogram recorded along a given urban bus trajectory. In this section, data are the measurements performed with a PolarX2 receiver from Septentrio. C/NO in the range of $35 \mathrm{dBHz}$ are representative of NLOS signals, in the range of 45 and more of LOS. From figure 2, the bimodal shape of the histogram allows us to assume that a classification of the LOS/NLOS satellite state of reception according to C/NO can be done even if the threshold between the two states is difficult to determine accurately. Indeed, attenuation depends on several very different criteria such as the receiver performance, the nature of the obstacles and the quality of the receiver. Here the threshold has been fixed equal to $40 \mathrm{~dB}$.

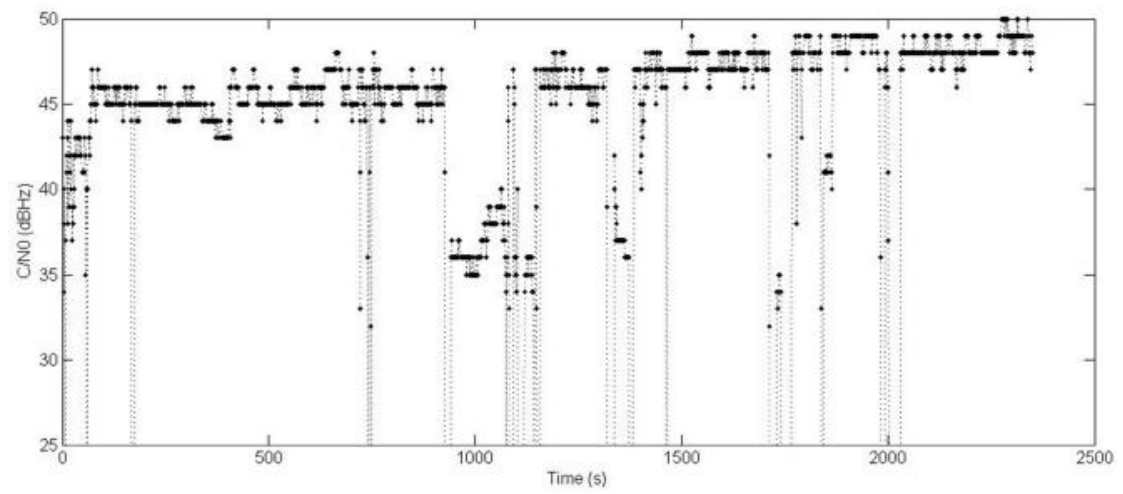

Fig. 1. C/NO variation versus time along a bus trajectory in an urban environment for satellite $n^{\circ} 10$. 


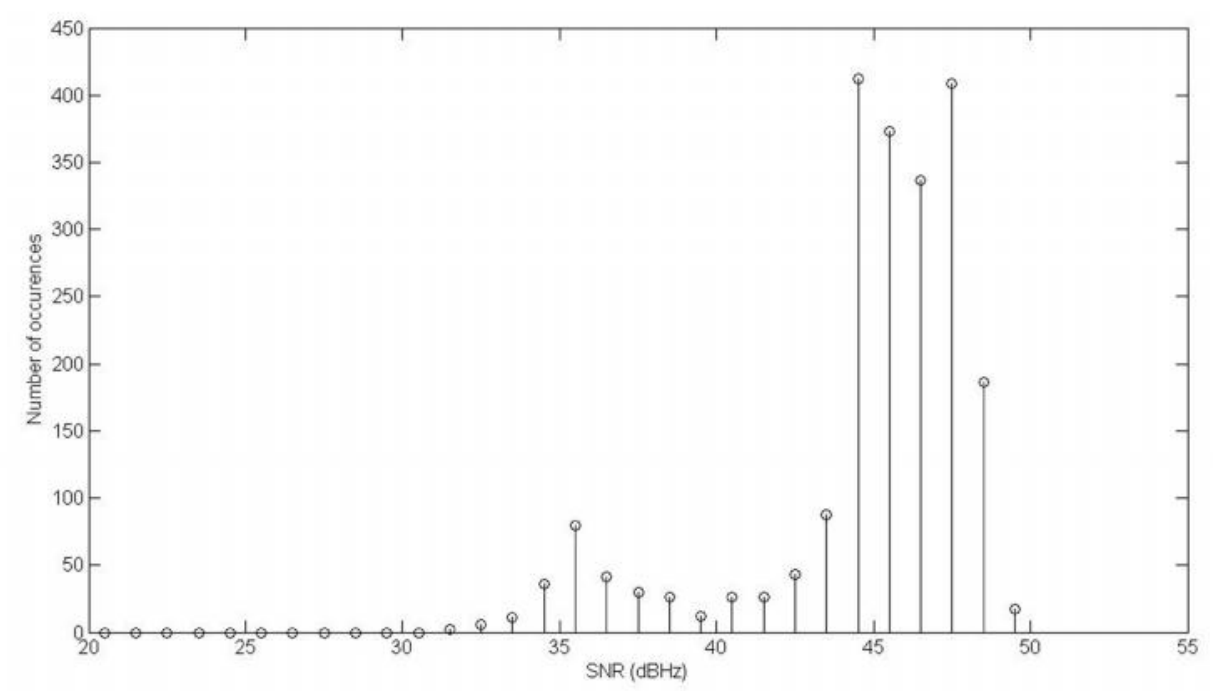

Fig. 2. C/NO histogram for one satellite along a bus trajectory in an urban environment for satellite $\mathrm{n}^{\circ} 10$

\subsection{NLOS state error distribution}

Once the state of reception determined, these reception states can be associated to pseudo range error distributions. As GNSS satellites move within time, the state of reception of a satellite $i$ varies versus time. Considering a large period of measurement allows the satellite to cover a large elevation variation as illustrated in figure 3. The longer the period of measurement, the bigger is the chance to encounter several error modes. Indeed during LOS periods, a centered Gaussian distribution is expected. In the NLOS periods, biased Gaussians, corresponding to the reflection characteristics will appear.

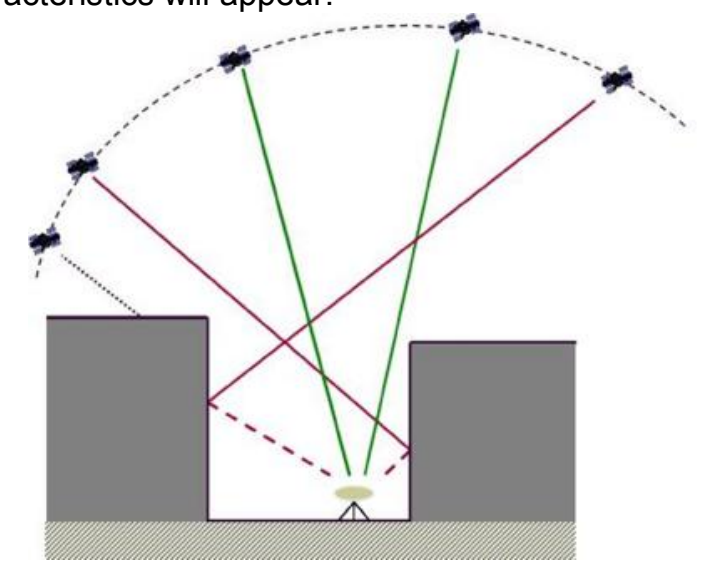

Fig. 3. Evolution of the reception states for one satellite versus time.

To illustrate the Gaussians evolution, the record of one given satellite pseudo ranges in a constricted area along a period of 300 minutes has been performed. A data acquisition was performed in a blue-sky environment (on the roof of the laboratory) where an obstacle was added. This obstacle is a metal plate which measures $1 \mathrm{~m}$ by $1 \mathrm{~m}$, placed at $40 \mathrm{~cm}$ from the antenna, with an inclination angle of $10^{\circ}$. The antenna is oriented northward. This operation procedure is used to maximize the signal reflections on the obstacle. For this experimentation, the equipment used is a Septentrio PolaRx2 receiver, bi-frequency and EGNOS-compatible. With such a receiver, the quality of propagation error corrections (tropospheric, ionospheric, etc) is high. Consequently, the residual pseudo range error is mainly dependent on the receiver noise, the clock errors and the potential delay due to signal reflections. Figure 4 presents the evolution of the density versus time when considering a time sliding window of 240s of observation (Viandier et al., 2010). 


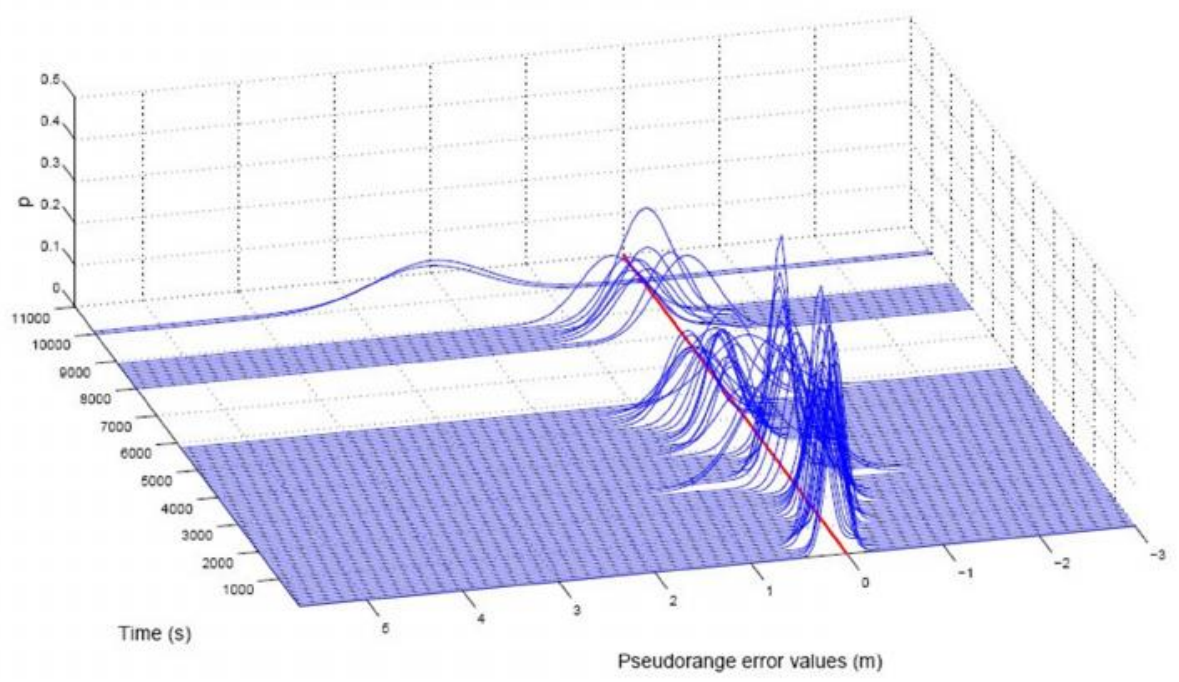

Fig. 4. Evolution of pseudo range error density versus time during a static acquisition.

Function of the period length, a Gaussian mixture modeling could be built to take into account all the distributions encountered as illustrated on figure 5.
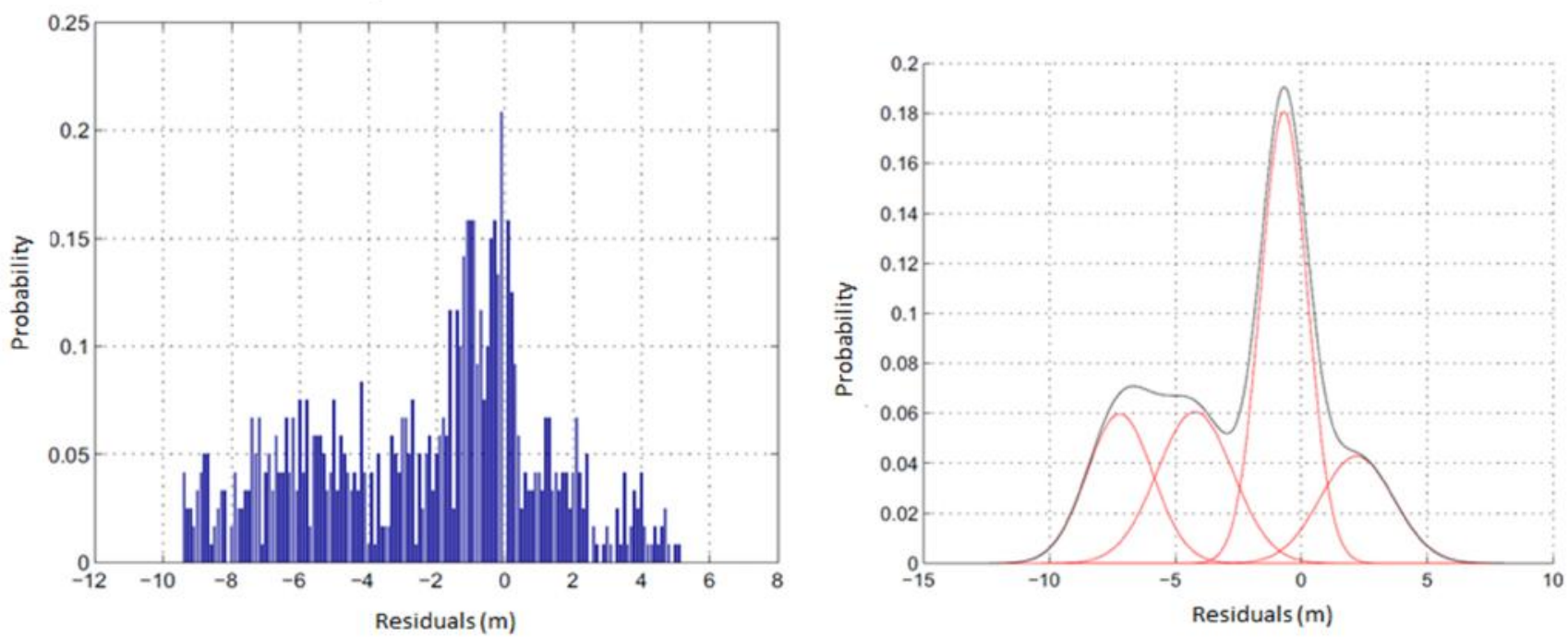

Fig. 5. Set of residuals acquired in a suburban area and its modeling by a Gaussian Mixture (here 4 Gaussian curves).

In a dynamic positioning process, where the environment of propagation around the vehicle constantly changes, observation duration of residuals is a parameter to adapt dynamically. The shorter the period will be, the more accurate will be the model for the specific environment. The duration could be linked to the vehicle speed. Indeed, the faster the vehicle moves, the faster the propagation environment changes. The change of propagation environment will induce switches in the satellite reception states and thus will modify the error density shape. On the contrary, a vehicle stop can be detected and used to enlarge the observation duration. According to the observation duration, changes caused by signal reflections will be close to changes in the Normal law parameters: mean and variance. However, this idea is not the purpose of the present paper and will not be developed here but is still under study.

In the next sections, we will work with temporal sliding windows. For each position at instant $t$, the error distribution will be modeled by a Gaussian mixture considering recent past pseudo range error measurements.

Next section will show how a Markov Chain will be used to take into account the temporal variation of reception states during a trip. 


\section{MARKOV CHAIN USE IN A JUMP MARKOV SYSTEM (JMS)}

\subsection{Markov chain definitions and properties}

Markov chains are simple and efficient models to simulate temporal series and many studies use this theory in the context of satellite transmissions (Perez-Fontan et al., 1997)(Arndt et al., 2012). Markov chains allow characterizing satellite propagation channel that has subjacent Markovian properties. Indeed, slow fading, caused by the changes of obstacles encountered by the signal, results in strong fluctuations of the mean level of the received power and can be seen as resulting from a Markov chain. Each state of the chain corresponds then to a particular propagation condition. These fluctuations can be modeled as probability transitions of the Markov Chain. The lines below summarize the principles of finite state first order discrete-time Markov chains.

Let $\mathbf{S}$ be a finite state space. A probability $p$ on $\mathbf{S}$ is a family $p=\left(p_{i} ; i \in \mathbf{S}\right)$ which verifies:

$$
0 \leq p_{i} \leq 1, i \in S \text { and } \sum_{i \in S} p_{i}=1
$$

A Discrete-Time Markov chain on $S$ is a sequence of random variables $\left\{X_{k}, k \geq 0\right\}$, which satisfies, for any $k$ instant and any series $i_{0}, \ldots, i_{k+1} \in S$ :

$$
P\left(X_{k+1}=i_{k+1} / X_{0}=i_{0}, \ldots, X_{k}=i_{k}\right)=P\left(X_{k+1}=i_{k+1} / X_{k}=i_{k}\right)
$$

The probabilities $P\left(X_{k+1}=j / X_{k}=i\right)=p_{i j}$ are known as the transition probabilities from state $i$ to state $j$.

Let $X=\left(X_{k}, k \geq 0\right)$ be a Markov chain on $S$. The probability distribution $p=\left(p_{i}, i \in S\right)$ defined by $p_{i}=P\left(X_{0}=i\right)$ is called the initial law of the Markov chain $X$.

The $M$ matrix which coefficients are the $p_{i j}$, $i$ and $j S$, is known as the Markov chain transition matrix.

The probability distribution of the chain $\left\{X_{k}\right\}$, with transition matrix $M$ and initial law $p$ is defined by:

$$
P\left(X_{0}=x_{0}, \ldots, X_{n}=x_{n}\right)=p_{x_{0}} p_{x_{0} x_{1}} \ldots p_{x_{n-1} x_{n}} \text {. }
$$

A Markov chain is completely defined by a transition matrix and an initial law. In the context of this paper, this Markov chain models the different possible states of reception. We show in the next section how it can be used in a new statistical filtering process to improve localization accuracy.

\subsection{JMS and particle filter}

A Jump Markov System is used here to select for each satellite reception state the best pseudo range error distribution to use in a particle filter based estimation process. We describe hereafter this algorithm. A Jump Markov System is a particular application of Switching State-Space Models. Its role is to switch between several observation models according to a current state variable. At a given time $t$, a model is thus defined by an evolution model and a measurement model among all the possible ones. In our case, we call $r_{t}$ the index variable defining the reception state at time $\mathrm{t}$. This variable pilots a Markovian evolution model $p\left(x_{t} / x_{t-1}, r_{t}\right)$ and the observation model $p\left(y_{t} / x_{t}, r_{t}\right)$. For example, in this study, $r_{t}$ can take $\mathrm{N}=3$ states defined at the beginning of this paper. The states at time $t$ are computed through a Markov chain matrix transition $M^{t}$ as represented in figure 6.

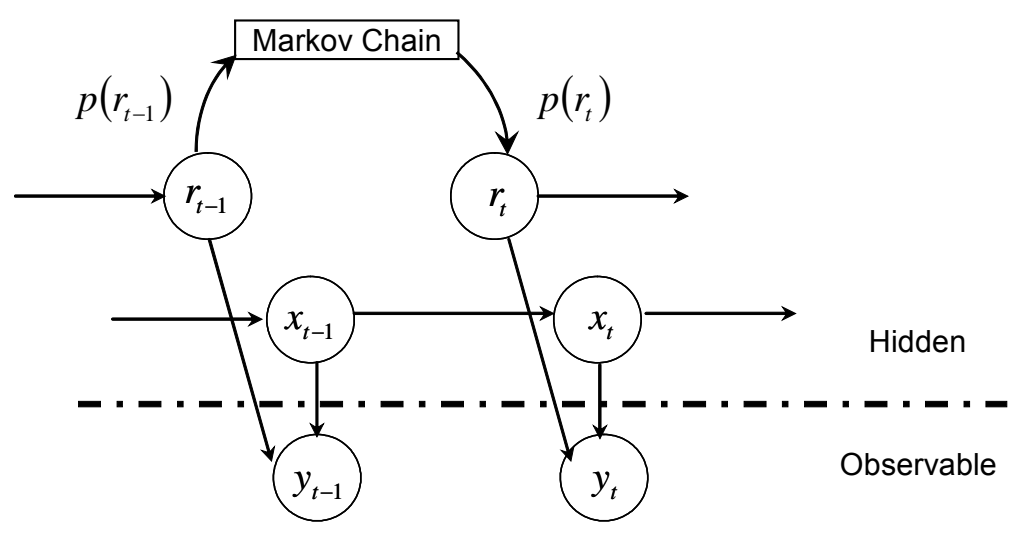

Fig. 6. Switching observation model for one satellite.

As the model becomes nonlinear and non-Gaussian, the Kalman filter is no more adapted. We chose a particle 
filter to estimate the state vector (position, bias, velocity and bias drift here). Indeed, particle filter allows managing both non Gaussian noises and nonlinear models. The filter principle consists in generating many samples drawn according to a probability distribution. The more the filter generates samples, the more the result is accurate, with the consequence that the algorithmic cost grows.

In the frame of nonlinear non Gaussian dynamical systems, the use of a particle filter relies on both observation and process time evolution models usually expressed as follows:

$x_{t}=f\left(x_{t-1}, r_{t}\right)+w_{t-1}$

$y_{t}=h\left(x_{t}, r_{t}\right)+v_{t}$

Here $x_{t}$ is the state vector at the time $\mathrm{t} ;{ }_{f}$ is the state transition function; $y_{t}$ is the measurements vector; $h$ is the measurement connection function; $w$ is the process noise and $v$ the measurement noise. Consequently, particle filter is therefore interesting here because $h$ is non-linear and $v$ has unspecified distribution.

\section{ApPLICATION TO GNSS URBAN LOCALIZATION}

\subsection{Markov Chain transition matrix}

Multi-sensors systems are considered in most of robotics GNSS applications. In a multi-sensor system, each of the sensors can face different modes of work: usually "nominal", "degraded" and "failure". In our context, the modes will depend on the environmental conditions as described in section 2. $r$ is an indicator of the sensor quality. As already said previously, the chosen model is here based on the Karasawa model (Karasawa et al., 1997) that considers a three state Markov chain.

Let us then consider each satellite $i$ as an autonomous sensor. $r_{t, i}$ represents the reception state at the time $t$ for the satellite $i$ :

$$
\begin{gathered}
r_{t, i}=j \text { where }{ }_{j \in\{0,1,2\}}(6) \\
\qquad \begin{array}{l}
r_{t, i}=0 \text { if the state is Blocked } \\
r_{t, i}=1 \text { if the state is LOS (direct or multipath). } \\
r_{t, i}=2 \text { if the state is NLOS. }
\end{array}
\end{gathered}
$$

In the blocked reception state the sensor is unavailable. In the nominal sensor state, the noise distribution is known as well as the nominal error model. And in presence of an alternate path state (NLOS), a distribution modeled by a Gaussian mixture will be applied.

To model the reception state of each satellite, a Markov Chain with three states will be defined for each satellite $i$. A transition matrix $M^{i}$ is associated to each satellite $i$ as well as a discrete Markov variable $r_{t, i}$ which represents the reception state at the time $t$ for the satellite $i$.

Let us now define:

$$
\alpha_{t, i}=\left[\begin{array}{l}
P\left(X_{t}=0\right) \\
P\left(X_{t}=1\right) \\
P\left(X_{t}=2\right)
\end{array}\right]
$$

Following the Markovian assumption, we can write:

$$
\alpha_{t, i}=M^{i} \alpha_{t-1, i}
$$

Here, $M^{i}$ is dependent on the environment of reception, being composed of the transition probabilities from one state to another. As was stated while modeling the distribution of errors, we consider that the propagation environment varies frequently along the trajectory. $M^{i}$ will then vary with respect to time along a sliding window. We 
will now write $M_{t}^{i}$ to emphasis this variation versus time. The last task is to estimate this matrix.

\subsection{Estimation of $M_{t}^{i}$}

We work with a sliding windows built upon the $n$ instants preceding the estimation time. M is computed for instant $t$ and satellite $i$ based on a frequentist approach. In the following, we forget the subscript $i$ for the sake of notation simplicity.

Let $N_{l, j}^{n}$ be the number of transitions from state / to $j$ during these $n$ instants: $N_{l, j}^{n}=\sum_{k=t-n}^{t} \delta\left(X_{k-1}=I, X_{k}=j\right)$

and let $N^{n}$ be the total number of state transition during the $n$ instants, then we have the following estimation:

$$
\hat{p}_{l, j}=\frac{N_{l, j}^{n}}{N^{n}} \text { and } \hat{M}=\left[\begin{array}{lll}
\hat{p}_{00} & \hat{p}_{01} & \hat{p}_{02} \\
\hat{p}_{10} & \hat{p}_{11} & \hat{p}_{12} \\
\hat{p}_{20} & \hat{p}_{21} & \hat{p}_{22}
\end{array}\right]
$$

\section{RESULTS}

Two types of data are used in this section. The first set is obtained by simulation. The main advantage is the control and explanation of every cause of error on the position. Two sets arise out of real measurements: one acquired in the city of Rouen, following a bus line crossing the city from east to west, interesting for the diversity of the crossed environments, and one in the city of Belfort, small old city center, interesting for the harsh reception of the signals.

\subsection{Results obtained from simulated data}

The algorithm described above has been first evaluated with ERGOSPACE ${ }^{\circledR}$ simulation data. ERGOSPACE ${ }^{\circledR}$ simulation software simulates electromagnetic signal propagation in 3D realistic environments. This software has been developed as a support for the development of GNSS applications. The deterministic method of Ray Tracing determines the possible paths of the rays received. The software provides output data files in a Matlab format, allowing extracting the transition matrices. Thanks to the simulation, the reception states of the satellites are known deterministically.

In the context of the French project ANGO, we have used a realistic 3D model of the city of Rouen, France, extracted from IGN ${ }^{1}$ databases. The test scenario follows an urban bus. Vehicle velocity is considered constant (equal to $50 \mathrm{~km} / \mathrm{h}$ ). Along the line $(8.25 \mathrm{~km}$ long, 594 measurements), the propagation environment is variable (wooden, urban and clear areas...) as illustrated in figure 7.

As detailed in previous sections, as the mobile travels through different types of environments, error properties and reception state probabilities are not constant along the line.

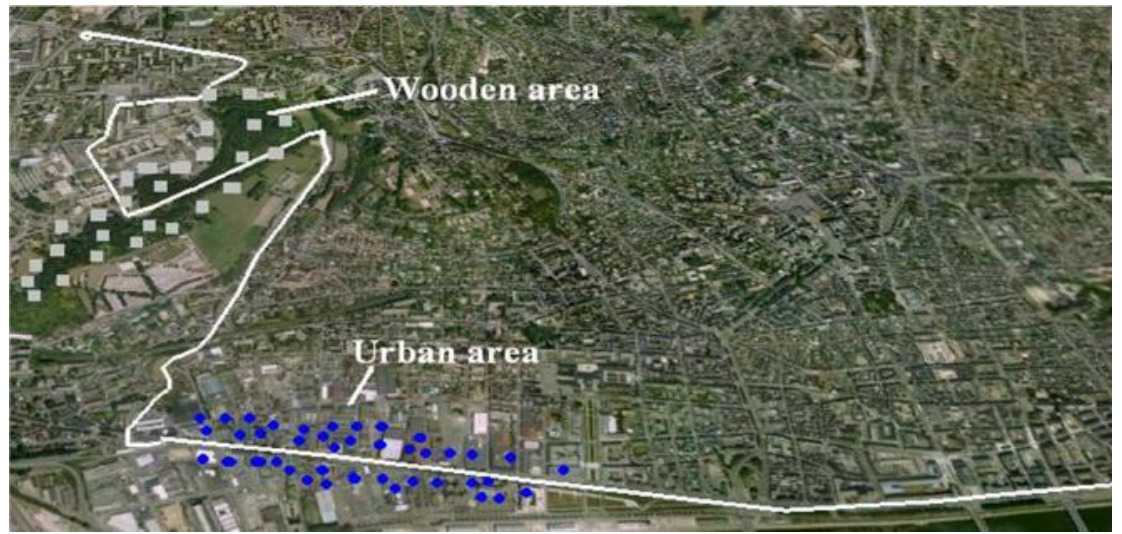

Fig. 7. Sky view of the bus line in Rouen.

With the simulation software characteristics, the measurement noises are reduced to the sum of a white Gaussian noise (mean 0 , variance 1) and geometrical delays caused by signal reflections on the surrounding obstacles. Common mode errors such as ionospheric, tropospheric, clock and ephemeris errors are corrected (Grewal et al., 
2001).

The input data used in ERGOSPACE are: the constellation almanacs; the 3D model of the environment; the vehicle trajectory; the start time of the run and the maximum number of reflections (3).

The geometrical biases caused by reflections are extracted from the results as inputs for our algorithm. The satellite positions and the exact geometrical distances between satellites and receiver are computed directly from the almanacs.

In the JMS, the PF uses 100 samples. As the observation windows are shorter than the windows of the figure 5 example, we have proposed an error model with only 2 Gaussians in the mixture. Results presented in the following correspond to one run of the vehicle. As the GPS satellites move versus time, slight differences in the values have been obtained for accuracy and other performance criteria.

\subsubsection{Satellite states of reception}

One of the main advantages working with simulation data is that every characteristic of the signal can be known. ERGOSPACE qualifies the number of reflections that affect each satellite signal, at each instant $t$. Figure 8 shows a comparison between the real states of reception (given by Ergospace) for satellite 27 in blue and the estimated states in red. Along this series of data, the beginning of the run travels through a "free of obstacle" environment and most of the time, the signal is received directly. When entering a more urbanized area (time 100), variations of the real state of reception go much faster. However, due to the sliding window principle (duration of 40 seconds), the chain requires 40 s to become efficient again and detect the alternate state, and rapid variations disappear when averaging. The duration of the sliding window has been chosen experimentally and corresponds to the mean duration of visibility of a large obstacle along an urban run of the vehicle. But future works will focus on dynamic enhancements to reduce this time of adaptation.

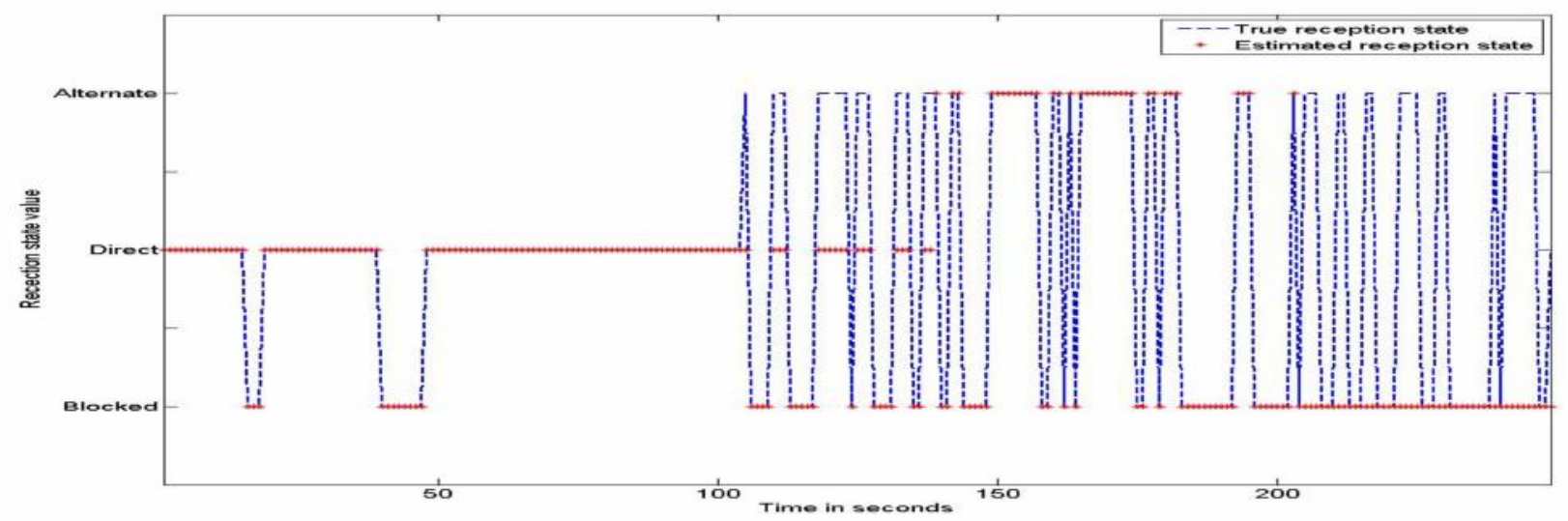

Fig. 8. Chronogram of reception states for one satellite along a trajectory.

\subsubsection{JMS Results with simulated data}

In order to evaluate the added-value of our algorithm, the localization performances of several algorithms are compared along the same path. Implemented methods are: our Jump Markov System (JMS), a classical Extended Kalman Filter (EKF) and a Particle Filter (PF). EKF is the most frequently implemented filter in recent receivers. EKF and PF assume that pseudo range error distributions are white and Gaussian.

Figure 9 draws the position error obtained with the three methods. The figure shows that the JMS algorithm is more accurate than classical methods like EKF filter. Moreover JMS error variation shows a more stable evolution than EKF error. In particular, EKF shows the biggest error peaks due to dynamic changes of the vehicle. PF reduces partially these errors but less than JMS that minimizes the observation model variations as well as vehicle dynamic changes.

Table I compares the previously described methods in terms of mean error and availability. In this table, availability is defined as the percentage of time during which the position is computed with accuracy better than $5 \mathrm{~m}$. Furthermore, the Mean Down Time (MDT) and Mean Up Time (MUT) have been mentioned. MDT indicates the time during which the service is unavailable, MUT the time of availability. MTBF indicates the mean time between two failure events, e.g. unavailability of the position or unacceptable accuracy. These are some of the reliability criteria defined in RAMS studies (Reliability, Availability, Maintainability and Safety) (Beugin., 2012) in particular used for railway applications. 


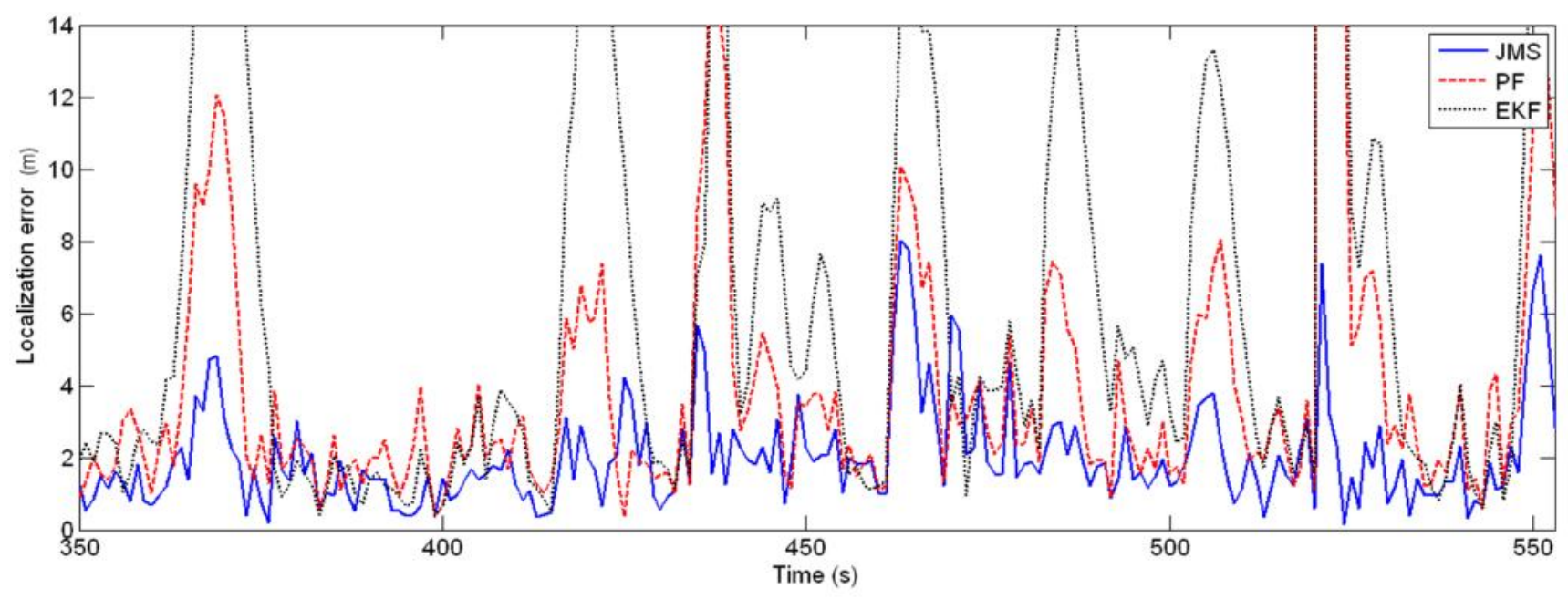

Fig. 9. Comparison of error variation along the time between Extended Kalman Filter and Jump Markov System

TABLE I

Comparison of the performances of the three algorithms: EKF, PF and our JMS based on simulated data

\begin{tabular}{|c|c|c|c|c|c|c|c|}
\hline & Mean error & $\begin{array}{c}\text { Min error X- } \\
\text { axis }\end{array}$ & $\begin{array}{c}\text { Min error Y- } \\
\text { axis }\end{array}$ & $\begin{array}{c}\text { Min error Z- } \\
\text { axis }\end{array}$ & MDT & $\begin{array}{c}\text { MUT } \\
\text { Availability } \\
(<5 \mathrm{~m})\end{array}$ \\
\hline EKF & $5.34 \mathrm{~m}$ & $3.07 \mathrm{~m}$ & $2.21 \mathrm{~m}$ & $2.97 \mathrm{~m}$ & $5.97 \mathrm{~s}$ & $11.16 \mathrm{~s}$ & $64.44 \%$ \\
\hline PF & $3.72 \mathrm{~m}$ & $2.07 \mathrm{~m}$ & $1.49 \mathrm{~m}$ & $2.07 \mathrm{~m}$ & $3.39 \mathrm{~s}$ & $13.87 \mathrm{~s}$ & $79.96 \%$ \\
\hline JMS & $2.65 \mathrm{~m}$ & $1.30 \mathrm{~m}$ & $0.88 \mathrm{~m}$ & $1.76 \mathrm{~m}$ & $2.50 \mathrm{~s}$ & $20.54 \mathrm{~s}$ & $89.17 \%$ \\
\hline
\end{tabular}

Table I highlights the performance improvements in terms of accuracy and availability of the positioning. Indeed, the mean error is divided by two and the number of points with an interesting accuracy (lower than $5 \mathrm{~m}$ ) increases from $64 \%$ to $89 \%$ of the time. In parallel, the MDT is reduced to $2.5 \mathrm{~s}$, corresponding to a lack of positioning information of $35 \mathrm{~m}$ length.

We can also notice that some of the erroneous points are easy to identify because of their unrealistic value compared to the previous ones (more than 300m farther). The implementation of fault detection process could easily exclude such points (Kuusniemi et al., 2004)(Wang et al., 2009).

\subsection{Results obtained from real data}

After a first evaluation with simulation data, this paragraph is devoted to some results obtained in two different environments with real data acquired from the bus line of Rouen previously described and a trajectory in the city of Belfort. The benefits along the Rouen line are modest because of the quite low density of obstacles along the Seine. Results obtained with the data recorded in the city of Belfort show more visibly the added-value of the method in a very constricted environment.

With both data, the parameters used for the JMS are the following: length of the observation windows 40 s, 2 Gaussians for each mixture and a number of particles equal to 50. The threshold for the distinction between LOS and NLOS state is fixed equal to $40 \mathrm{~dB}$. The errors are estimated by an orthogonal projection of the measurements on the trajectory of reference.

\subsubsection{Results of the Rouen measurement campaign}

During the ANGO project, measurements have been performed with a Septentrio PolarX2 receiver. Table II compares two positioning solutions that apply respectively a classical EKF and our JMS. 1300 points are measured, one per second. The mean error is almost equal with the two solutions. That can be explained by the quality of the receiver, the quality of the raw data used and because the solution proposed changes the noise in NLOS cases only. If the occurrence of NLOS is low, the impact will be low too. But we can observe that the percentage of points provided with an accuracy lower than $3 \mathrm{~m}$ and $5 \mathrm{~m}$ increases with our method. Our solution enhances availability and the length of usability of the GNSS solution. 
Expert Systems with Applications, Volume 40, Issue 15, 1 November 2013, Pages 5956-5964 http://dx.doi.org/10.1016/j.eswa.2013.05.026 OElsevier

TABLE II

Comparison of the performances of the three algorithms: EKF and our JMS based on real data from Rouen

\begin{tabular}{|c|c|c|c|c|}
\hline & \multirow[b]{2}{*}{ Mean error } & \multicolumn{3}{|c|}{ Availability of the solution for specific accuracy } \\
\hline & & $<3 \mathrm{~m}$ & $<5 \mathrm{~m}$ & $<10 \mathrm{~m}$ \\
\hline EKF & $6.9 \mathrm{~m}$ & $43 \%$ & $65 \%$ & - \\
\hline JMS (GM) & $6.65 \mathrm{~m}$ & $53.19 \%$ & $70.94 \%$ & $82.65 \%$ \\
\hline
\end{tabular}

\subsubsection{Results of the Belfort measurement campaign}

Raw GPS measurements have been recorded thanks to the CAPLOC project in the city of Belfort. The receiver used is a cheaper equipment than previously. It is composed of an uBlox chip implemented in a SafeDrive unit. The environment in Belfort is more constricted than Rouen. Indeed, measurements show a mean accuracy of the positioning solution of $14.85 \mathrm{~m}$ along a trajectory in the more dense area of the city center where streets are particularly narrows. 2000 points are measured, one per second.

Table III summarizes the performances of the positioning algorithm for a $2 \mathrm{D}$ computation. We compare the output of the receiver (Typical EKF solution without any specific filtering process on pseudo ranges) and our JMS solution. These results show a better improvement of the mean accuracy thanks to the JMS algorithm, mainly because the solution proposed is particularly adapted to such an environment. Indeed, in this dense urban area, the error distribution is far away from a Gaussian centered law as soon as the signal is reflected.

TABLE III

Comparison of the performances of the three algorithms: EKF and our JMS based on real data from Belfort

\begin{tabular}{|c|c|c|c|c|}
\hline & \multirow[b]{2}{*}{ Mean error } & \multicolumn{3}{|c|}{ Availability of the solution for specific accuracy } \\
\hline & & $<3 \mathrm{~m}$ & $<5 \mathrm{~m}$ & $<10 \mathrm{~m}$ \\
\hline Receiver sol. & $14.85 \mathrm{~m}$ & $10 \%$ & $30 \%$ & $56 \%$ \\
\hline JMS (GM) & $10.50 \mathrm{~m}$ & $18.21 \%$ & $41.96 \%$ & $71.05 \%$ \\
\hline
\end{tabular}

\subsubsection{Discussion about real time implementation}

At this stage this method cannot be implemented in real time. Indeed, we focus in this study on statistical modelling and improvement of the estimation process. No work has been done on the algorithmic nor the implementation (we use Matlab). We are aware that specific studies still remain to be done mainly for the particle filter implementation and the transition matrix estimation. But this is out of the scope of this paper.

The choice of the number of Gaussian is also a problem as well as the choice of the duration of the sliding window which introduce inertia in the estimation process. Selection models techniques could then be used but this would not tackle the inertia problem. As an alternative, we could also build models that adapt automatically the number of Gaussian to data. This can be done using Bayesian non-parametric models like Dirichlet Process Mixture (DPM) (Rabaoui et al., 2009 and Rabaoui et al., 2012). Such a method brings a solution to both problems but increases the computational cost because of the use of Monte Carlo Markov Chain (MCMC) within the estimation process. The problem is however similar to the one introduced by the particle filtering and could therefore be the purpose of a same study.

\section{CONCLUSION}

In order to enhance the accuracy of GNSS solutions, this paper proposes a filtering-based process that takes into account the noise bias caused by signal propagation delays in harsh environments. It also allows to include biased measurements in the computation of the positioning solution instead of excluding them. The goal of the solution is to provide the best accuracy and availability from a GPS receiver alone in presence of 4 signals, even if they are received in NLOS conditions. Other sensors can then enhance our solution in order to face remaining unavailability periods.

The main contribution of this paper is to show how to model pseudo range errors in the case of reflected signals and how to use them in a Jump Markov System to enhance accuracy and availability of a simple GNSS solution. The algorithm has been validated on simulated data representative of a real bus line trajectory of the city of Rouen, France and running through different types of environments. The application of the algorithm on real measured data has validated the conclusions obtained with the simulation. The use of a sliding window considering last measurements for estimating the noise of the recent past has shown that the method was adaptable with different 
Expert Systems with Applications, Volume 40, Issue 15, 1 November 2013, Pages 5956-5964

http://dx.doi.org/10.1016/j.eswa.2013.05.026 OElsevier

scenarios and different obstacle natures. It has also shown that, whatever the starting time is, availability of the JMS solution is always better than the other filter tested. Its main drawback seems to be its sensitivity to some of the changes of the observation state that the Markov chain cannot detect.

Future works will focus on more accurate estimating error distribution using Dirichlet Process Mixture (DPM) and on testing our algorithms in real time in order to mitigate the rapid errors identified in this study and allow the system to be more independent of historical data. DPM provides a sequential density estimation of pseudo range errors that adapt automatically the model order to the available data.

\section{ACKNOWLEDGMENT}

The authors want to thank the French programs ANR, PREDIT, CISIT and the DRI for supporting the projects and work mentioned in this paper.

\section{REFERENCES}

Arndt, D., Ihlow, A., Heyn, T., Heuberger, A., Prieto-Cerdeira, R.,, Eberlein, E., 2012. State modelling of the land mobile propagation channel for dual-satellite systems. EURASIP Journal on Wireless Communications and Networking, 2012(1), 1-21.

Beugin, J., Marais, J., 2012. Simulation-based evaluation of dependability and safety properties of satellite technologies for railway localization, Transportation Research Part C: Emerging Technologies, Volume 22, June 2012, Pages 42-57.

Brissaud,A., Boschetti, M., et al., Comparing autonomous GPS, A-GPS, Long Term Ephemeris aided GPS, DGPS and RTK, ENC-GNSS 2007, Geneva, Switzerland, May 29 - June 1, 2007, 10p

Dempster, A.P., Laird, N.M., Rubin, D.B., 1977. Maximum Likelihood from Incomplete Data with the EM Algorithm (with discussion). Journal of the Royal Statistical Society, Series B.

Grewal, M.S., Weill, L.R., Andrews, A.P., 2001. Global Positioning Systems, Inertial Navigation and Integration. John Wiley \& Sons, USA.

E.D. Kaplan (Ed), 1996. Understanding GPS, principles and applications.

Jiang, Z., Groves, P. D., 2012. NLOS GPS signal detection using a dual-polarisation antenna. GPS Solutions, 1-12.

Karasawa, Y., Kimura, K., Minamisono, K., 1997. Analysis of Availability Improvement in LMSS by Means of Satellite Diversity Based on ThreeState Propagation Channel Model. IEEE Trans. On Vehicular Technology, Vol. 46, No 4, Nov. 1997

Kovář, P., Vejražka, F., Seidl, L., Puričer, P., Assessment of the EGNOS Signal Availability for Land Mobile User. 12th Saint Petersburg International Conference on Integrated Navigation Systems. Saint Petersburgh 2005, May 23 - 25, 2005, s. 148 - 156

Kuusniemi, H., Lachapelle, G., GNSS signal Reliability Testing in urban and indoor environments, Proceedings of the ION NTM Conference, San Diego, CA, January 26-28, 2004.

Lentmaier, M., Krach, B., Robertson, P., 2008. Bayesian Time Delay Estimation of GNSS Signals in Dynamic Multipath Environments. Hindawi Publishing Corporation, International Journal of Navigation and Observation, Vol. 2008, Article ID 372651, 11 pages.

Marais, J., Pouyet, V., 2007. Lateral guidance and longitudinal control for bus rapid transit- Focus on the positioning system based on GPS and RFID technology, ITS World Congress, Oct. 2007, Beijing.

Nahimana, D.F., Marais, J., Duflos, E., 2007. A Jump Markov System for modelling a realistic error model depending on satellite reception state in urban environment. Proceedings of ION GNSS 2007, Institute of Navigation, 7p.

Obst, M., Schubert, R., Streiter, R., \& Liberto, C. 2011. Benefit Analysis of EGNOS/EDAS for Urban Road Transport Applications. Proceedings of the 8th ITS European Congress.

Ochieng, W.Y. Sauer, K., 2002. Urban road transport navigation: performance of the global positioning system after selective availability. Transportation research part C 10, 171-187, Pergamon

Perez-Fontán, F, González, J.P., Ferreiro, J.J.S., Castro, M.A.V., Buonomo, S., Baptista, J.P., 1997. Complex envelope three-state markov model based simulator for the narrow-band LMS channel. International Journal of Satellite Communications Volume 15, Issue 1, 1-15, John Wiley \& Sons, Ltd.

Pérez Fontán, F., Vázquez-Castro, M., Enjamio Cabado, C., Pita Garciá, J., Kubista, E., 2001. Statistical modeling of the LMS channel. IEEE Transactions on Vehicular Technology, Viol. 50, No. 6, November 2001, 1549-1567.

Pervan, B.S., Lawrence, D.G., Cohen, C.E., Parkinson, B.W., 1996. Parity space methods for autonomous fault detection and exclusion using GPS carrier phase. Position Location and Navigation Symposium, 1996, IEEE 1996 Volume , Issue , 22-26 Apr 1996, 649 - 656

Quddus, M.A., 2006, High Integrity Map Matching Algorithms for Advanced Transport Telematics Applications, PhD, Imperial College London.

Rabaoui, A., Viandier, N., Marais, J., Duflos, E., 2009. Using Dirichlet Process Mixtures for the Modelling of GNSS Pseudorange Errors in Urban Canyon. ION-GNSS 2009, Savannah, Georgia, September 22-25, 2009, 9p

Rabaoui A., Viandier N.,Marais J., Duflos E., Vanheeghe P., 2012. Dirichlet Process Mixtures for Density Estimation in Dynamic Nonlinear

Modeling: Application to GPS Positioning in Urban Canyons, IEEE Transactions on Signal Processing Journal, Vol. 60(4), April 2012, pp 1638-

1665.

Santa, J., et al., 2009. An analysis of communications and navigation issues in collision avoidance support systems. Transport. Res. Part C, doi: 10.1016/j.trc.2009.05.007

Scalise, S., Lücke, O., Vilchez Torralbo, E. 2006. A Link Availability Channel Model for the Railroad Satellite Channel, 24th AIAA International Communications Satellite Systems Conference (ICSSC), 11 - 14 June 2006, San Diego, California

Schubert, R., Mattern, N., Wanielik, G., 2008. An evaluation of nonlinear filtering algorithms for integrating GNSS and inertial measurements. Position, Location and Navigation Symposium, 2008 IEEE/ION Volume, Issue, 5-8 May 2008, 25 - 29

Viandier, N., Nahimana, D.F., Marais, J., Duflos, E., 2008. GNSS performance enhancement in urban environment based on pseudo-range error model, Proceedings of Position, Location and Navigation Symposium (PLANS) IEEE/ION 2008. Page(s):377 - 382. 6-8 Mai 2008. Monterey, California.

Viandier, N., 2009. Méthodes de validation des modèles d'erreurs de pseudodistances en fonction de l'environnement de propagation, Actes des journées des doctorants SPI de l'INRETS, Villeneuve d'Ascq, Avril 2009.

Viandier, N., Rabaoui, A., Marais, J., Duflos, E., 2010. Studies on DPM for the density estimation of pseudorange noises and evaluations on real data, IEEE PLANS, Palm Springs, -6-9 May, 2010.

Wang, J.-H., Gao, Y., 2007. High-Sensitivity GPS Data Classification Based on Signal Degradation Conditions. IEEE Transactions on Vehicular Technology, Volume 56, Issue 2, March 2007, 566 - 574

Wang, J., Ober, P.B., On the availability of fault detection and exclusion in GNSS receiver autonomous integrity monitoring, The Journal of Navigation, 62, 251-261, 2009. 
Expert Systems with Applications, Volume 40, Issue 15, 1 November 2013, Pages 5956-5964 http://dx.doi.org/10.1016/j.eswa.2013.05.026 OElsevier

Wang, S. Sand, Analysis of multipath mitigation techniques with Land Mobile Satellite channel model, Radioengineering, Vol. 21, № 4 , Dec 2012 , pp1067-1077.

Wieser, A., Gaggl, M., Hartinger, H., 2005. Improved Positioning Accuracy with High-Sensitivity GNSS Receivers and SNR Aided Integrity Monitoring of Pseudo-Range Observations. Proc. of ION GNSS 18th International Technical Meeting of the Satellite Division, Long Beach.

Youngsun, Y., Ho Yun, D.K., Kee, C., 2008. A Gaussian Sum filter approach for DGNSS integrity monitoring. The Journal of Navigation, vol. 61, 2008, 687-703. 